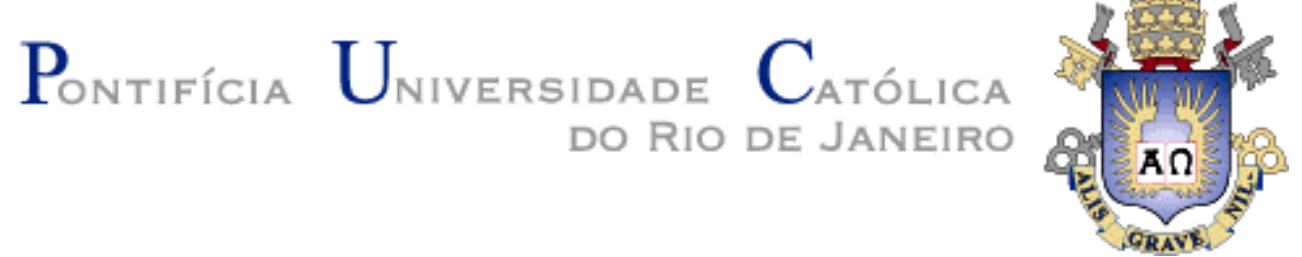

Isela Macía Bertrán

\title{
Avaliação da Qualidade de Software com Base em Modelos UML
}

Dissertação de Mestrado

Dissertação apresentada como requisito parcial para a obtenção do título de Mestre pelo Programa de Pós-graduação em Informática da PUC-Rio.

Orientadores: Prof. Arndt von Staa

Cláudio Nogueira Sant'Anna 
Avaliação da Qualidade de Software com Base em Modelos UML

Dissertação apresentada como requisito parcial para obtenção do título de Mestre pelo Programa de PósGraduação em Informática da PUC-Rio. Aprovada pela Comissão Examinadora abaixo assinada.

Prof. Arndt von Staa

Orientador

Departamento de Informática - PUC-Rio

Prof. Carlos José Pereira de Lucena

Departamento de Informática - PUC-Rio

Prof.- Simone Diniz Junqueira Barbosa

Departamento de Informática - PUC-Rio

Prof. José Eugenio Leal

Coordenador Setorial do Centro Técnico Científico - PUC-Rio

Rio de Janeiro, 25 de Março de 2009 
Todos os direitos reservados. É proibida a reprodução total ou parcial do trabalho sem autorização da universidade, da autora e do orientador.

\section{Isela Macía Bertrán}

Graduou-se em Ciência da Computação na Universidade de Havana (UH) em 2006. Atuou como desenvolvedor de software até 2007.

Ficha Catalográfica

\section{Macía Bertrán, Isela}

Avaliação da qualidade de software com base em modelos UML / Isela Macía Bertrán ; orientadores: Arndt Von Staa, Cláudio Nogueira Sant'Anna. - 2009.

131 f. : il. ; $30 \mathrm{~cm}$

Dissertação (Mestrado em Informática)Pontifícia Universidade Católica do Rio de Janeiro, Rio de Janeiro, 2009.

Inclui bibliografia

1. Informática - Teses. 2. Design de software orientado a objetos. 3. Diagramas de classe. 4. Métricas de software. 5. Estratégias de detecção de bad smells. 6. Modelos da qualidade. 7. Engenharia de software experimental. I. Staa, Arndt von. II. Sant'Anna, Cláudio Nogueira. III. Pontifícia Universidade Católica do Rio de Janeiro. Departamento de Informática. IV. Título.

CDD: 004 


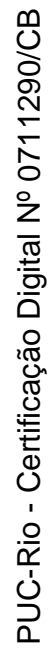

Aos meus pais 


\section{Agradecimentos}

Meus agradecimentos mais especiais vão para as pessoas que julgo mais responsáveis por tudo que alcancei até aqui: meus maravilhosos pais. Muito obrigada pelo amor, confiança, educação e uma vida inteira de dedicação que, sem dúvida, tem sido essenciais na minha formação e luta pelos meus objetivos. Ao resto de minha família, pelo carinho, apoio e momentos de alegria sempre importantes, ainda mais na distância: à minha irmã, avô, tias, tios e primos.

Ilimitados agradecimentos a Julio pela maneira única que me ama e compreende dia a dia. Por sua paciência e incentivo em meus momentos de dúvidas e temores. Sou muito grata e orgulhosa de tê-lo ao meu lado.

Agradeço especialmente ao meu orientador, professor Arndt von Staa pois, sem seu apoio, este trabalho não teria sido possível. Ele me deu liberdade para escolher o tema deste trabalho, indicando-me sempre o caminho correto. Agradeço por terme mostrado o mundo das métricas e da qualidade de software.

Ao meu co-orientador, Cláudio Sant'Anna, pela orientação, dicas, parceria e dedicação. Você foi fundamental para o meu trabalho, sempre fornecendo comentários e sugestões para a melhoria da qualidade do conteúdo e da redação de minha dissertação.

Aos colegas do LES e do departamento de informática, pelos momentos compartilhados e aprendizado dividido. Agradeço especialmente a Andrew por ter-me facilitado parte dos sistemas analisados nesta dissertação e a Camila por sua ajuda na confecção de minhas primeiras apresentações.

Aos amigos Francisco, Frank, Ilaria, Evelin e Dunieskys pelo companheirismo e momentos que passamos juntos.

A Merlin, Yuanersi, Yahima, Ivan, grandes amigos de longa data, e cuja amizade sempre se mantive forte apesar da distância. 
Agradeço, finalmente, a CAPES pelo apoio financeiro.

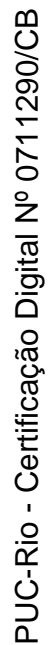




\section{Resumo}

Macía, Isela. Avaliação da Qualidade de Software com Base em Modelos UML. Rio de Janeiro, 2009. 131p. Dissertação de Mestrado - Departamento de Informática, Pontifícia Universidade Católica do Rio de Janeiro.

Um dos objetivos da engenharia de software é a construção de software com um nível de qualidade elevado com o menor custo e no menor tempo possível. Nesse contexto, muitas técnicas para o controle da qualidade de design de software têm sido definidas. Além disso, mecanismos baseados em métricas para a detecção de problemas também têm sido definidos. A maioria dessas técnicas e mecanismos foca a análise do código fonte. Porém, para reduzir retrabalho inútil, é importante utilizar técnicas de análise da qualidade capazes de detectar problemas de design já desde os modelos dos sistemas. Esta dissertação propõe: (i) um conjunto de estratégias de detecção para identificar, em modelos UML, problemas de design específicos e recorrentes na literatura: Long Parameter List, God Class, Data Class, Shotgun Surgery, Misplaced Class e God Package, e (ii) a utilização do modelo da qualidade QMOOD para avaliar design de software a partir de seus diagramas de classes. Para automatizar a aplicação destes mecanismos foi implementada uma ferramenta: a QCDTool. Os mecanismos desenvolvidos foram avaliados no contexto de dois estudos experimentais. O primeiro estudo avaliou a acurácia, precisão e recall das estratégias de detecção propostas. Esse estudo mostrou os benefícios e desvantagens da aplicação, em modelos, das estratégias de detecção propostas. O segundo estudo avaliou a utilidade da aplicação do modelo da qualidade QMOOD em diagramas UML. Esse estudo mostrou que foi possível identificar, em diagramas de classes, variações das propriedades de design, e, conseqüentemente, dos atributos da qualidade nos sistemas analisados.

\section{Palavras-chave}

Design de software orientado a objetos, diagramas de classe, métricas de software, estratégias de detecção de bad smells, modelos da qualidade, engenharia de software experimental. 


\section{Abstract}

Macía, Isela. Evaluation of Software Quality Based on UML Models. Rio de Janeiro, 2009. 131p. MSc. Dissertation - Departamento de Informática, Pontifícia Universidade Católica do Rio de Janeiro.

One of the goals of software engineering is the development of high quality software at a small cost an in a short period of time. In this context, several techniques have been defined for controlling the quality of software designs. Furthermore, many metrics-based mechanisms have been defined for detecting software design flaws. Most of these mechanisms and techniques focus on analyzing the source code. However, in order to reduce unnecessary rework it is important to use quality analysis techniques that allow the detection of design flaws earlier in the development cycle. We believe that these techniques should analyze design flaws starting from software models. This dissertation proposes: (i) a set of strategies to detect, in UML models, specific and recurrent design problems: Long Parameter List, God Class, Data Class, Shotgun Surgery, Misplaced Class and God Package; (ii) and the use of QMOOD quality model to analyze class diagrams. To automate the application of these mechanisms we implemented a tool: the QCDTool. The detection strategies and QMOOD model were evaluated in the context of two experimental studies. The first study analyzed the accuracy, precision and recall of the proposed detection strategies. The second study analyzed the utility of use QMOOD quality model in the class diagrams. The results of the first study have shown the benefits and drawbacks of the application in class diagrams of some of the proposed detection strategies. The second study shows that it was possible to identify, based on class diagrams, variations of the design properties and consequently, of the quality attributes in the analyzed systems.

\section{Keywords}

Object oriented software design, class diagrams, software metrics, bad smells detection strategies, quality models, experimental software engineering. 


\section{Sumário}

1 Introdução

2 Avaliação Quantitativa do Design de Software 19

2.1. Design de Software Orientado a Objetos 19

2.2. Medição de Software 22

2.3. Modelos de Controle da Qualidade de Design 00

2.3.1. Modelos de Qualidade 25

2.3.1.1. Modelo de Qualidade QMOOD 26

2.3.2. Estratégias para a Detecção de Problemas de Design 31

3 Trabalhos Relacionados $\quad 35$

3.1. Métricas para Diagramas de Classes 36

3.2. Detecção de Problemas de Design no Código Fonte 38

3.3. Análise de Design em Diagramas UML 39

4 Detecção de Problemas de Design 42

4.1. Características dos Valores Limites 43

4.2. Problemas de Design para Classes 43

4.2.1. Data Class 43

4.2.1.1. Estratégia de Detecção para Código Fonte 44

4.2.1.2. Estratégia de Detecção para Modelo 46

4.2.2. God Class $\quad 47$

4.2.2.1. Estratégia para Código Fonte 48

4.2.2.2. Estratégia de Detecção para Modelo 49

4.2.3. Shotgun Surgery $\quad 51$

4.2.3.1. Estratégia de Detecção para Código Fonte 51

4.2.3.2. Estratégia de Detecção para Modelo 52

4.3. Problemas de Design em Pacotes 54

4.3.1. God Package $\quad 54$

4.3.1.1. Estratégia de Detecção para Código Fonte 55 
4.3.2. Misplaced Class 58

4.3.2.1. Estratégia de Detecção para Código Fonte 58

4.3.2.2. Estratégia de Detecção para Modelo 60

4.4. Problemas de Design para Métodos 61

4.4.1. Long Parameter List 61

5 QCDTool: Uma Ferramenta para Avaliar a Qualidade do Design em Modelos 63

5.1. Visão Geral da Ferramenta 63

5.2. Processo de Funcionamento 64

5.3. Arquitetura e Implementação 65

5.3.1. Módulo de Importação de Diagramas de Classes 66

5.3.2. Módulo de Aplicação das Métricas 68

5.3.3. Aplicação das Estratégias de Detecção 69

5.3.4. Aplicação dos Modelos da Qualidade 73

5.3.5. GUI 75

5.3.6. Exportação dos Resultados 77

5.4. Pontos Fixos e Pontos de Extensão 78

5.5. Instanciação 79

5.5.1. Importação dos Diagramas 80

5.5.2. Definição de Métricas 81

5.5.3. Definição de Estratégias de Detecção 82

5.5.4. Definição de Modelos da Qualidade 83

5.5.5. Exportação dos Resultados da Análise 84

6 Estudos Experimentais $\quad 85$

6.1. Avaliação das Estratégias de Detecção 86

6.1.1. Sistemas Envolvidos 86

6.1.2. Estrutura do Estudo 88

6.1.2.1. Definição do Estudo 88

6.1.2.2. Planejamento do Estudo 88

6.1.2.3. Operação 90

6.1.3. Resultados 91 
6.1.3.1. Resultados para a Estratégia de Detecção Data Class 92

6.1.3.2. Resultados para a Estratégia de Detecção God Class 94

6.1.3.3. Resultados para a Estratégia de Detecção Shotgun Surgery 96

6.1.3.4. Resultados para a Estratégia de Detecção God Package 98

6.1.3.5. Resultados para a Estratégia de Detecção Misplaced Class 100

6.1.3.6. Resultados para a Estratégia de Detecção Long Parameter $\begin{array}{ll}\text { List } & 101\end{array}$

6.1.4. Discussões sobre os Resultados 102

6.2. Avaliação do modelo da qualidade QMOOD 103

$\begin{array}{ll}\text { 6.2.1. O Formato do Estudo } & 104\end{array}$

6.2.2. Resultados das Propriedades do Design e Atributos da

$\begin{array}{ll}\text { Qualidade Avaliados } & 105\end{array}$

$\begin{array}{ll}\text { 6.2.3. Conclusões do Estudo } & 108\end{array}$

$\begin{array}{ll}\text { 6.3. Ameaças à Validade dos Estudos Realizados } & 109\end{array}$

7 Conclusões e Trabalhos Futuros $\quad 111$

$\begin{array}{ll}\text { 7.1. Contribuições } & 111\end{array}$

$\begin{array}{ll}\text { 7.2. Trabalhos Futuros } & 113\end{array}$

8 Referências $\quad 115$

Anexo A - Document Type Definition do arquivo XML utilizado por $\begin{array}{lr}\text { QCDTool } & 119\end{array}$

Anexo B - Documento gerado por QCDTool para Armazenar os Resultados das Estratégias de Detecção

Anexo C - Resultados da Aplicação das Estratégias de Detecção no Primeiro Estudo Experimental 


\section{Lista de figuras}

Figura 1 - Níveis e relações no QMOOD. 27

Figura 2 - Mecanismo de filtragem e composição. 33

Figura 3 - Estratégia de detecção de Data Class para código. 45

Figura 4 - Estratégia de detecção de Data Class para modelo. 47

Figura 5 - Estratégia de detecção de God Class para código. 48

Figura 6 - Estratégia de detecção de God Class para modelo. 50

Figura 7 - Estratégia de detecção de Shotgun Surgery para código. 51

Figura 8 - Estratégia de detecção de Shotgun Surgery para modelo. 53

Figura 9 - Estratégia de detecção de God Package para código. 55

Figura 10 - Estratégia de detecção de God Package para modelo. 57

Figura 11 - Estratégia de detecção de Misplaced Class para código. 59

Figura 12 - Estratégia de detecção de Misplaced Class para modelo. $\quad 60$

Figura 13 - Estratégia de detecção de Long Parameter List. 62

Figura 14 - Processo de funcionamento da ferramenta. 64

Figura 15 - Diagrama de módulos da ferramenta. $\quad 65$

Figura 16 - Atividades da importação dos diagramas de classes. 66

Figura 17 - Módulo de importação dos diagramas. 68

Figura 18 - Representação das métricas. $\quad 69$

Figura 19 - Representação das estratégias de detecção. $\quad 70$

Figura 20 - Representação do mecanismo de filtragem. 72

$\begin{array}{ll}\text { Figura } 21 \text { - Estrutura dos operadores. } & 73\end{array}$

Figura 22 - Modelos da qualidade. 75

Figura 23 - QDCTool: (a) treeview com classes e pacotes; (b) tabela com resultados das métricas associadas a uma componente; (c) tabela com resultados das propriedades de design. 76

Figura 24 - Relações de dependência, herança e generalização entre as $\begin{array}{ll}\text { classes. } & 76\end{array}$

Figura 25 - Componentes de design classificados como problemáticos. $\quad 77$

Figura 26 - Estrutura do módulo de exportação dos resultados. 78

Figura 27 - Instanciação do módulo de importação dos diagramas. 80 
Figura 28 - Instanciação das métricas.

Figura 29 - Instanciação das estratégias de detecção. 83

Figura 30 - Instanciação do modelo QMOOD.

Figura 31 - Instanciação do módulo de exportação. 


\section{Lista de tabelas}

Tabela 1 - Métricas do modelo QMOOD. 28

Tabela 2 - Relações entre as métricas e as propriedades do design. 30

Tabela 3 - Classificação dos filtros para dados. 32

Tabela 4 - Métricas para diagramas de classes. 36

Tabela 5 - Instanciação da ferramenta. $\quad 79$

Tabela 6 - Características dos sistemas envolvidos no estudo. 88

Tabela 7 - Resultados da estratégia de detecção Data Class. 93

Tabela 8 - Resultados para a estratégia de detecção God Class. 95

Tabela 9 - Resultados para a estratégia de detecção Shotgun Surgery. 96

Tabela 10 - Resultados para a estratégia de detecção God Package. 99

Tabela 11 - Resultados para a estratégia de detecção Misplaced Class. 100

Tabela 12 - Resultados para a estratégia de detecção de Long Parameter

$\begin{array}{ll}\text { List. } & 101\end{array}$

Tabela 13 - Valores das propriedades de design. 105

Tabela 14 - Valores normalizados das propriedades de design. 106

Tabela 15 - Resultados da quantificação dos atributos da qualidade. 108

Tabela 16 - Resultados da estratégia de detecção Long Parameter List 122

Tabela 17 - Resultados das estratégias de detecção Data Class 124

Tabela 18 - Resultados da estratégia de detecção God Class. 126

Tabela 19 - Resultados da estratégia de detecção Shotgun Surgery. 127

Tabela 20 - Resultados da estratégia de detecção God Package. 130

Tabela 21 - Resultados da estratégia de detecção Misplaced Class. 131 


\section{Lista de Acrônimos e Abreviações}

ATFD - Access To Foreign Data.

CA - Changing Attributes.

CAM - Coesion Among Methods.

CC - Client Class.

CL - Class Locality.

CM - Changing Methods.

DD - Depency Dispersion.

DTD - Document Type Definition.

GQM - Goal-Question-Metric.

GUI - Graphical User Interface.

MOF - MetaObject Facility.

NOAM - Number Of Access Methods.

NOCC - Number Of Client Class.

NOCP - Number Of Client Packages.

NOED - Number Of External Dependencies.

NOP - Number Of Parameters.

NOPA - Number Of Public Attributes.

OMG - Object Management Group.

OO - Orientado a Objetos.

PC - Package Cohesion.

PS - Package Size.

QMOOD - Quality Model Object Oriented Design.

SAX - Simple API XML.

TCC - Tight Class Cohesion.

UML - Unified Modeling Language.

WMC - Weighted Method per Class.

WOC - Weight Of Class.

XMI - XML Metadata Interchange.

XML - Extensible Markup Language. 